\title{
On value distribution and uniqueness of meromorphic function with finite logarithmic order concerning its derivative and $q$-shift difference
}

\section{Xiu-Min Zheng ${ }^{1 *}$ and Hong-Yan Xu ${ }^{2}$}

*Correspondence:

zhengxiumin2008@sina.com 1 Institute of Mathematics and Information Science, Jiangxi Normal University, Nanchang, 330022, China Full list of author information is available at the end of the article

\begin{abstract}
In this paper, we study the value distribution of a meromorphic function $f(z)$ concerning its derivative $f^{\prime}(z)$ and $q$-shift difference $f(q z+c)$, where $f(z)$ is of finite logarithmic order. We also investigate the uniqueness of differential- $q$-shift-difference polynomials with more general forms of entire functions of order zero.
\end{abstract}

Keywords: differential-q-shift-difference; meromorphic function; logarithmic order

\section{Introduction and main results}

The fundamental theorems and the standard notations of the Nevanlinna value distribution theory of meromorphic functions will be used (see e.g. Hayman [1], Yang [2] and Yi and Yang [3]). In addition, for a meromorphic function $f(z)$, we use $S(r, f)$ to denote any quantity satisfying $S(r, f)=o(T(r, f))$ for all $r$ outside a possible exceptional set $E$ of finite logarithmic measure $\lim _{r \rightarrow \infty} \int_{(1, r] \cap E} \frac{d t}{t}<\infty$ and also use $S_{1}(r, f)$ to denote any quantity satisfying $S_{1}(r, f)=o(T(r, f))$ for all $r$ on a set $F$ of logarithmic density 1 , where the logarithmic density of a set $F$ is defined by

$$
\lim _{r \rightarrow \infty} \frac{1}{\log r} \int_{(1, r] \cap F} \frac{1}{t} d t
$$

The order of a meromorphic function $f(z)$ is defined by

$$
\rho(f)=\limsup _{r \rightarrow \infty} \frac{\log T(r, f)}{\log r}
$$

The logarithmic order of a meromorphic function $f(z)$ is defined by (see [4])

$$
\rho_{\log }(f)=\limsup _{r \rightarrow \infty} \frac{\log T(r, f)}{\log \log r}
$$

If $\rho_{\log }(f)<\infty$, then $f(z)$ is said to be of finite logarithmic order. It is clear that if a meromorphic function $f(z)$ has finite logarithmic order, then $f(z)$ has order zero.

O2014 Zheng and Xu; licensee Springer. This is an Open Access article distributed under the terms of the Creative Commons Attribution License (http://creativecommons.org/licenses/by/2.0), which permits unrestricted use, distribution, and reproduction in any medium, provided the original work is properly cited. 
Remark 1.1 $[4,5]$ If $f(z)$ is a meromorphic function of finite positive logarithmic order $\rho_{\log }(f)$, then $T(r, f)$ has proximate logarithmic order $\rho_{\log }(f)$. The logarithmic-type function of $T(r, f)$ is defined as $U(r, f)=(\log r)^{\rho_{\log }(f)}$. We have $T(r, f) \leq U(r, f)$ for sufficiently large $r$. The logarithmic exponent of convergence of $a$-points of $f(z)$ is equal to the logarithmic order of $n\left(r, \frac{1}{f-a}\right)$, which is defined as

$$
\lambda_{\log }(a)=\limsup _{r \rightarrow \infty} \frac{\log n\left(r, \frac{1}{f-a}\right)}{\log \log r} .
$$

We see by [4] that for a meromorphic function $f(z)$ of finite positive logarithmic order $\rho_{\log }(f)$, the logarithmic order of $N\left(r, \frac{1}{f-a}\right)$ is $\lambda_{\log }(a)+1$, where $\lambda_{\log }(a)$ is the logarithmic order of $n\left(r, \frac{1}{f-a}\right)$.

Moreover, we assume in the whole paper that $m, n, k, t_{m}, t_{n}$ are positive integers, $q \in$ $C \backslash\{0\}, c \in C$, and $a(z)$ is a non-zero small function with respect to $f(z)$, that is, $a(z)$ is a non-zero meromorphic function of growth $S(r, f)$.

Many mathematicians were interested in the value distribution of different expressions of meromorphic functions and obtained lots of important theorems (see e.g. [1, 6-8]). Especially, Hayman [7] discussed Picard's values of meromorphic functions and their derivatives, and he obtained the following famous theorem in 1959.

Theorem 1.1 [7] Let $f(z)$ be a transcendental entire function. Then

(a) for $n \geq 2, f^{\prime}(z) f(z)^{n}$ assumes all finite values except possibly zero infinitely often;

(b) for $n \geq 3$ and $a \neq 0, f^{\prime}(z)-a f(z)^{n}$ assumes all finite values infinitely often.

Further, for a transcendental meromorphic function $f(z)$, Chen and Fang [9] obtained the following result.

Theorem 1.2 [9, Theorem 1] Let $f(z)$ be a transcendental meromorphic function. If $n \geq 1$ is a positive integer, $f^{\prime}(z) f(z)^{n}-1$ has infinitely many zeros.

Recently, with the establishments of difference analogies of the Nevanlinna theory (see e.g. [10-12]), many mathematicians focused on studying difference analogies of Theorems 1.1 and 1.2. The main purpose of these results (see e.g. $[5,13-16]$ ) is to get the sharp estimation of the value of $n$ to make difference polynomials $f(z+c) f(z)^{n}-a$ and $f(z+c)-a f(z)^{n}-b$ admit infinitely many zeros.

Meantime, $q$-difference analogies of the Nevanlinna theory and their applications on the value distribution of $q$-difference polynomials and $q$-shift-difference equations are also studied (see e.g. [17-19]). Especially, for a transcendental meromorphic (resp. entire) function $f(z)$ of order zero, Zhang and Korhonen [20] studied the value distribution of $q$ difference polynomials of $f(z)$ and found that if $n \geq 6$ (resp. $n \geq 2$ ), then $f(q z) f(z)^{n}$ assumes every non-zero value $a \in C$ infinitely often (see [20, Theorem 4.1]).

Further, $\mathrm{Xu}$ and Zhang [21] investigated the zeros of $q$-shift difference polynomials of meromorphic functions of finite logarithmic order and obtained the following result in 2012 .

Theorem 1.3 [21, Theorem 2.1] Iff $(z)$ is a transcendental meromorphic function of finite logarithmic order $\rho_{\log }(f)$, with the logarithmic exponent of convergence of poles less than 
$\rho_{\log }(f)-1$ and $q, c$ are non-zero complex constants, then for $n \geq 2, f(z)^{n} f(q z+c)$ assumes every value $b \in C$ infinitely often.

One main aim of this paper is to investigate the zeros of differential- $q$-shift-difference polynomials about $f(z), f^{\prime}(z)$, and $f(q z+c)$, where $f(z)$ is of finite positive logarithmic order.

Theorem 1.4 Let $f(z)$ be a transcendental meromorphic function of finite logarithmic or$\operatorname{der} \rho_{\mathrm{log}}(f)$, with the logarithmic exponent of convergence of poles less than $\rho_{\mathrm{log}}(f)-1$. Set

$$
F_{1}(z)=f(q z+c)^{n} f^{\prime}(z)
$$

If $n \geq 3$, then $F_{1}(z)-a(z)$ has infinitely many zeros.

We also deal with the value distribution of a differential- $q$-shift-difference polynomial with another form about $f(z), f^{\prime}(z)$ and $f(q z+c)$, and we obtain the following result.

Theorem 1.5 Let $f(z)$ be a transcendental meromorphic function of finite logarithmic or$\operatorname{der} \rho_{\log }(f)$, with the logarithmic exponent of convergence of poles less than $\rho_{\mathrm{log}}(f)-1$. Set

$$
F_{2}(z)=f(q z+c)^{n}+f^{\prime}(z)+f(z) .
$$

If $n \geq 3$, then $F_{2}(z)-a$ has infinitely many zeros, where $a \in C$.

Some more general differential- $q$-shift-difference polynomials are investigated in the following.

Theorem 1.6 Let $f(z)$ be a transcendental meromorphic function of finite logarithmic or$\operatorname{der} \rho_{\log }(f)$, with the logarithmic exponent of convergence of poles less than $\rho_{\log }(f)-1$. Set

$$
F_{3}(z)=f(z)^{m} f(q z+c)^{n} f^{\prime}(z)
$$

If $m, n$ satisfy $m \geq n+2$ or $n \geq m+2$, then $F_{3}(z)-a(z)$ has infinitely many zeros.

Let

$$
P_{n}(z)=a_{n} z^{n}+a_{n-1} z^{n-1}+\cdots+a_{1} z+a_{0}
$$

be a non-zero polynomial, where $a_{0}, a_{1}, \ldots, a_{n}(\neq 0)$ are complex constants and $t_{n}$ is the number of the distinct zeros of $P_{n}(z)$. Then we also obtain the following results.

Theorem 1.7 Let $f(z)$ be a transcendental meromorphic function of finite logarithmic or$\operatorname{der} \rho_{\log }(f)$, with the logarithmic exponent of convergence of poles less than $\rho_{\log }(f)-1$. Set

$$
F_{4}(z)=f(z)^{m} P_{n}(f(q z+c)) \prod_{j=1}^{k} f^{(j)}(z) .
$$

If $m \geq n+k+1$, then $F_{4}(z)-a(z)$ has infinitely many zeros. 
Theorem 1.8 Let $f(z)$ be a transcendental meromorphic function of finite logarithmic or$\operatorname{der} \rho_{\log }(f)$, with the logarithmic exponent of convergence of poles less than $\rho_{\log }(f)-1$. Set

$$
F_{5}(z)=P_{m}(f(z)) f(q z+c)^{n} \prod_{j=1}^{k} f^{(j)}(z) .
$$

If $m \geq n+k+1$, then $F_{5}(z)-a(z)$ has infinitely many zeros.

Next, we investigate the uniqueness of differential- $q$-shift-difference polynomials of entire functions of order zero and obtain the following results.

Theorem 1.9 Let $f(z)$ and $g(z)$ be transcendental entire functions of order zero and $n \geq 5$. If $f(q z+c)^{n} f^{\prime}(z)$ and $g(q z+c)^{n} g^{\prime}(z)$ share a non-zero polynomial $p(z) C M$, then $f(q z+$ $c)^{n} f^{\prime}(z)=g(q z+c)^{n} g^{\prime}(z)$.

Theorem 1.10 Let $f(z)$ and $g(z)$ be transcendental entire functions of order zero and $m \geq$ $n+2 t_{n}+5 . \operatorname{Iff}(z)^{m} P_{n}(f(q z+c)) f^{\prime}(z)$ and $g(z)^{m} P_{n}(g(q z+c)) g^{\prime}(z)$ share a non-zero polynomial $p(z) C M$, then $f(z)^{m} P_{n}(f(q z+c)) f^{\prime}(z)=g(z)^{m} P_{n}(g(q z+c)) g^{\prime}(z)$.

Theorem 1.11 Let $f(z)$ and $g(z)$ be transcendental entire functions of order zero and $n \geq m+2 t_{m}+5$. If $P_{m}(f(z)) f(q z+c)^{n} f^{\prime}(z)$ and $P_{m}(g(z)) g(q z+c)^{n} g^{\prime}(z)$ share a non-zero polynomial $p(z) C M$, then $P_{m}(f(z)) f(q z+c)^{n} f^{\prime}(z)=P_{m}(g(z)) g(q z+c)^{n} g^{\prime}(z)$.

\section{Some lemmas}

To prove the above theorems, we need some lemmas as follows.

Lemma 2.1 [3] Let $f(z)$ be a non-constant meromorphic function and $P(f)=a_{0}+a_{1} f+$ $\cdots+a_{n} f^{n}$, where $a_{0}, a_{1}, \ldots, a_{n}$ are complex constants and $a_{n} \neq 0$, then

$$
T(r, P(f))=n T(r, f)+S(r, f) .
$$

Lemma 2.2 [21] Let $f(z)$ be a transcendental meromorphic function of finite logarithmic order and $q, \eta$ be two non-zero complex constants. Then we have

$$
\begin{aligned}
& T(r, f(q z+\eta))=T(r, f)+S_{1}(r, f), \\
& N(r, f(q z+\eta))=N(r, f)+S_{1}(r, f), \quad N\left(r, \frac{1}{f(q z+\eta)}\right)=N\left(r, \frac{1}{f}\right)+S_{1}(r, f) .
\end{aligned}
$$

Lemma 2.3 [22, Theorem 2.1] Let $f(z)$ be a non-constant zero-order meromorphic function and $q \in C \backslash\{0\}$. Then

$$
m\left(r, \frac{f(q z+\eta)}{f(z)}\right)=S_{1}(r, f) .
$$

Lemma 2.4 [3, p.37] Let $f(z)$ be a non-constant meromorphic function in the complex plane and $l$ be a positive integer. Then

$$
T\left(r, f^{(l)}\right) \leq T(r, f)+l \bar{N}(r, f)+S(r, f), \quad N\left(r, f^{(l)}\right)=N(r, f)+l \bar{N}(r, f) .
$$


Lemma 2.5 [4] If $f(z)$ is a transcendental meromorphic function of finite logarithmic order $\rho_{\log }(f)$, then for any two distinct small functions $a(z)$ and $b(z)$ with respect to $f(z)$, we have

$$
T(r, f) \leq N\left(r, \frac{1}{f-a}\right)+N\left(r, \frac{1}{f-b}\right)+o(U(r, f))
$$

where $U(r, f)=(\log r)^{\rho_{\log }(f)}$ is a logarithmic-type function of $T(r, f)$. Furthermore, if $T(r, f)$ has a finite lower logarithmic order

$$
\mu=\liminf _{r \rightarrow \infty} \frac{\log T(r, f)}{\log \log r},
$$

with $\rho_{\log }(f)-\mu<1$, then

$$
T(r, f) \leq N\left(r, \frac{1}{f-a}\right)+N\left(r, \frac{1}{f-b}\right)+o(T(r, f)) .
$$

Remark 2.1 From the proof of Lemma 2.5 (see [4, Theorem 7.1]), we can easily see that complex values $a$ and $b$ can be changed into $a(z)$ and $b(z)$, where $a(z)$ and $b(z)$ are two distinct small functions with respect to $f(z)$.

Lemma 2.6 Let $f(z)$ be a transcendental meromorphic function of order zero, $F_{1}(z)=f(q z+$ $c)^{n} f^{\prime}(z)$. Then we have

$$
\begin{aligned}
(n-2) T(r, f)+S_{1}(r, f) & \leq(n-1) T(r, f)-N(r, f)+S_{1}(r, f) \\
& \leq T\left(r, F_{1}\right) \leq(n+2) T(r, f)+S_{1}(r, f) .
\end{aligned}
$$

Proof If $f(z)$ is a meromorphic function of order zero, from Lemmas 2.2 and 2.4, we have

$$
T\left(r, F_{1}\right) \leq n T(r, f(q z+c))+T\left(r, f^{\prime}\right) \leq(n+2) T(r, f)+S_{1}(r, f) .
$$

On the other hand, from Lemmas 2.2 and 2.4 again, we have

$$
\begin{aligned}
(n+1) T(r, f) & =T\left(r, f(q z+c)^{n+1}\right)+S_{1}(r, f) \\
& \leq T\left(r, F_{1}\right)+T\left(r, \frac{f(q z+c)}{f^{\prime}(z)}\right)+S_{1}(r, f) \\
& \leq T\left(r, F_{1}\right)+2 T(r, f)+N(r, f)+S_{1}(r, f) \\
& \leq T\left(r, F_{1}\right)+3 T(r, f)+S_{1}(r, f) .
\end{aligned}
$$

Thus, we get (1).

Lemma 2.7 Let $f(z)$ be a transcendental meromorphic function of zero order, $F_{3}(z)=$ $f(z)^{m} f(q z+c)^{n} f^{\prime}(z)$. Then we have

$$
T\left(r, F_{3}\right) \leq(m+n+2) T(r, f)+S_{1}(r, f)
$$


and

$$
T\left(r, F_{3}\right) \geq|m-n| T(r, f)-N(r, f)+S_{1}(r, f) \geq(|m-n|-1) T(r, f)+S_{1}(r, f) .
$$

Proof If $f(z)$ is a meromorphic function of order zero, from Lemmas 2.2 and 2.4, we have

$$
T\left(r, F_{3}\right) \leq m T(r, f)+n T(r, f(q z+c))+T\left(r, f^{\prime}\right) \leq(m+n+2) T(r, f)+S_{1}(r, f),
$$

that is, we have (2). On the other hand, from Lemmas 2.2 and 2.4, we have

$$
\begin{aligned}
(n+m+1) T(r, f) & =T\left(r, f^{n+m+1}\right)=T\left(r, \frac{f(z)^{n+1} F_{3}(z)}{f(q z+c)^{n} f^{\prime}(z)}\right) \\
& \leq T\left(r, F_{3}\right)+T\left(r, \frac{f}{f^{\prime}}\right)+T\left(r, \frac{f(z)^{n}}{f(q z+c)^{n}}\right) \\
& \leq T\left(r, F_{3}\right)+(2 n+1) T(r, f)+N(r, f)+S_{1}(r, f) \\
& \leq T\left(r, F_{3}\right)+(2 n+2) T(r, f)+S_{1}(r, f),
\end{aligned}
$$

that is, we have (3), where we assume $m \geq n$ without loss of generality.

Lemma 2.8 Let $f(z)$ be a transcendental meromorphic function of order zero, $F_{4}(z)=$ $f(z)^{m} P_{n}(f(q z+c)) \prod_{j=1}^{k} f^{(j)}(z)$. Then we have

$$
\begin{aligned}
& (m-n-k) T(r, f) \leq T\left(r, F_{4}\right)+\frac{k(k+1)}{2} N(r, f)+S_{1}(r, f), \\
& T\left(r, F_{4}\right) \leq\left(m+n+\frac{k(k+3)}{2}\right) T(r, f)+S_{1}(r, f) .
\end{aligned}
$$

Proof Since $f(z)$ is a transcendental meromorphic function of order zero, by Lemmas 2.1, 2.2 , and 2.4, we can easily get the second inequality. On the other hand, it follows by Lemmas $2.1,2.2$, and 2.4 that

$$
\begin{aligned}
(m+k) T(r, f) & =T\left(r, f^{m+k}\right) \leq T\left(r, \frac{f(z)^{k} F_{4}(z)}{P_{n}(f(q z+c)) \prod_{j=1}^{k} f^{(j)}(z)}\right) \\
& \leq T\left(r, F_{4}\right)+T\left(r, P_{n}(f(q z+c))\right)+T\left(r, \prod_{j=1}^{k} \frac{f^{(j)}}{f}\right) \\
& \leq T\left(r, F_{4}\right)+(n+2 k) T(r, f)+\frac{k(k+1)}{2} N(r, f)+S_{1}(r, f) .
\end{aligned}
$$

Thus, this completes the proof of Lemma 2.8 .

Similar to Lemma 2.8, we have the following lemma.

Lemma 2.9 Let $f(z)$ be a transcendental meromorphic function of zero order, $F_{5}(z)=$ $P_{m}(f(z)) f(q z+c)^{n} \prod_{j=1}^{k} f^{(j)}(z)$. Then we have

$$
\begin{aligned}
& (n-m-k) T(r, f) \leq T\left(r, F_{5}\right)+\frac{k(k+1)}{2} N(r, f)+S_{1}(r, f), \\
& T\left(r, F_{5}\right) \leq\left(m+n+\frac{k(k+3)}{2}\right) T(r, f)+S_{1}(r, f) .
\end{aligned}
$$




\section{Proofs of Theorems 1.4-1.8}

\subsection{Proof of Theorem 1.4}

It follows by Lemma 2.6 that $T\left(r, F_{1}\right)=O(T(r, f))$ holds for all $r$ on a set of logarithmic density 1 . Since $f(z)$ is transcendental and $n \geq 3, F_{1}(z)$ is transcendental by Lemma 2.6 again. Since the logarithmic exponent of convergence of poles of $f(z)$ less than $\rho_{\log }(f)-1$, we have

$$
\limsup _{r \rightarrow \infty} \frac{\log N(r, f)}{\log \log r}<\rho_{\log }(f) .
$$

Assume that $F_{1}(z)-a(z)$ has only finitely many zeros. Thus, by Lemmas 2.2, 2.4-2.6, we have

$$
\begin{aligned}
(n-2) T(r, f) & \leq T\left(r, F_{1}\right)+S_{1}(r, f) \\
& \leq N\left(r, F_{1}\right)+N\left(r, \frac{1}{F_{1}-a}\right)+o(U(r, f))+S_{1}(r, f) \\
& =N\left(r, f(q z+c)^{n} f^{\prime}(z)\right)+N\left(r, \frac{1}{F_{1}-a}\right)+o(U(r, f))+S_{1}(r, f) \\
& \leq(n+2) N(r, f)+N\left(r, \frac{1}{F_{1}-a}\right)+o(U(r, f))+S_{1}(r, f) .
\end{aligned}
$$

Thus, it follows that

$$
(n-2) T(r, f) \leq(n+2) N(r, f)+o(U(r, f))+S_{1}(r, f) .
$$

Since $n \geq 3$, the above inequality implies

$$
\limsup _{r \rightarrow \infty} \frac{\log T(r, f)}{\log \log r} \leq \limsup _{r \rightarrow \infty} \frac{\log N(r, f)}{\log \log r}<\rho_{\log }(f),
$$

which contradicts the fact that $T(r, f)$ has finite logarithmic order $\rho_{\log }(f)$. Thus, $F_{1}(z)-a(z)$ has infinitely many zeros, that is, $f(q z+c)^{n} f^{\prime}(z)-a(z)$ has infinitely many zeros.

This completes the proof of Theorem 1.4.

\subsection{Proof of Theorem 1.5}

Since $f(z)$ is a transcendental meromorphic function of finite logarithmic order, we first claim that $f^{\prime}(z)+f(z)-a \not \equiv 0$. In fact, if $f^{\prime}(z)+f(z)-a \equiv 0$, that is, $\frac{f^{\prime}(z)}{f(z)-a} \equiv-1$. By solving the above equation, we have $f(z)=A e^{-z}+a$, where $A$ is a non-zero complex constant. Thus, we have $\rho(f)=1$, which contradicts the fact that $f(z)$ is of order zero. Thus, set

$$
F(z)=\frac{a-f(z)-f^{\prime}(z)}{f(q z+c)^{n}} .
$$

It follows by Lemmas 2.2 and 2.4 that

$$
\begin{aligned}
n T(r, f) & =T\left(r, f(q z+c)^{n}\right)+S_{1}(r, f) \\
& \leq T\left(r, \frac{F(z)}{a-f(z)-f^{\prime}(z)}\right)+S_{1}(r, f)
\end{aligned}
$$




$$
\begin{aligned}
& \leq T(r, F)+T(r, f)+T\left(r, f^{\prime}\right)+S_{1}(r, f) \\
& \leq T(r, F)+2 T(r, f)+N(r, f)+S_{1}(r, f),
\end{aligned}
$$

that is,

$$
(n-2) T(r, f) \leq T(r, F)+N(r, f)+S_{1}(r, f) .
$$

On the other hand, we can easily get

$$
T(r, F) \leq T\left(r, f(q z+c)^{n}\right)+T(r, f)+T\left(r, f^{\prime}\right)+O(1) \leq(n+3) T(r, f)+S_{1}(r, f) .
$$

And it follows from (5) and $n \geq 3$ that

$$
T(r, F)=O(T(r, f))
$$

holds for all $r$ on a set of logarithmic density 1. By Lemma 2.2, we have

$$
\begin{aligned}
N\left(r, \frac{1}{F-1}\right) & =N\left(r, \frac{f(q z+c)^{n}}{f(q z+c)^{n}+f^{\prime}(z)+f(z)-a}\right) \\
& \leq N\left(r, \frac{1}{f(q z+c)^{n}+f^{\prime}(z)+f(z)-a}\right)+n N(r, f)+S_{1}(r, f) .
\end{aligned}
$$

Assume that $f(q z+c)^{n}+f^{\prime}(z)+f(z)-a$ has finitely many zeros, then

$$
N\left(r, \frac{1}{F-1}\right) \leq n N(r, f)+S_{1}(r, f)
$$

Since the logarithmic exponent of convergence of poles of $f(z)$ is less than $\rho_{\log }(f)-1$, we have

$$
\limsup _{r \rightarrow \infty} \frac{\log N(r, f)}{\log \log r}<\rho_{\log }(f) .
$$

Then, by Lemmas $2.4,2.5$, and (6), we have

$$
\begin{aligned}
T(r, F) & \leq N\left(r, \frac{1}{F}\right)+N\left(r, \frac{1}{F-1}\right)+o(U(r, f)) \\
& \leq N\left(r, \frac{1}{a-f-f^{\prime}}\right)+2 n N(r, f)+S_{1}(r, f)+o(U(r, f)) \\
& \leq 2 T(r, f)+(2 n+1) N(r, f)+S_{1}(r, f)+o(U(r, f)) .
\end{aligned}
$$

It follows by the above inequality and (4) that

$$
(n-4) T(r, f) \leq(2 n+2) N(r, f)+S_{1}(r, f)+o(U(r, f)) .
$$

Since $n \geq 5$, the above inequality implies

$$
\limsup _{r \rightarrow \infty} \frac{\log T(r, f)}{\log \log r} \leq \limsup _{r \rightarrow \infty} \frac{\log N(r, f)}{\log \log r}<\rho_{\log }(f),
$$


which contradicts that $T(r, f)$ has finite logarithmic order $\rho_{\log }(f)$. Thus, $f(q z+c)^{n}+f^{\prime}(z)+$ $f(z)-a$ has infinitely many zeros.

This completes the proof of Theorem 1.5.

\subsection{Proofs of Theorems 1.6, 1.7, and 1.8}

Similar to the argument as in Theorem 1.4, by applying Lemmas 2.7, 2.8, and 2.9 instead, we can easily prove Theorems $1.6,1.7$, and 1.8 respectively.

\section{Proofs of Theorems 1.9-1.11}

Here, we only give the proof of Theorem 1.10 because the methods of the proofs of Theorems $1.9,1.10$, and 1.11 are very similar.

\subsection{Proof of Theorem 1.10}

Denote

$$
F(z)=f(z)^{m} P_{n}(f(q z+c)) f^{\prime}(z), \quad G(z)=g(z)^{m} P_{n}(g(q z+c)) g^{\prime}(z) .
$$

Since $f(z)$ is a transcendental entire function of order zero, by Lemmas 2.1, 2.2, and 2.4, we have

$$
\begin{aligned}
T(r, F) & \leq T\left(r, f^{m}\right)+T\left(r, P_{n}(f(q z+c))+T\left(r, f^{\prime}\right)+O(1)\right. \\
& \leq(n+m+1) T(r, f)+S_{1}(r, f),
\end{aligned}
$$

and

$$
\begin{aligned}
(m+1) T(r, f) & =T\left(r, f^{m+1}\right)=T\left(r, \frac{f(z) F(z)}{P_{n}(f(q z+c)) f^{\prime}(z)}\right) \\
& \leq T(r, F)+T\left(r, \frac{f}{f^{\prime}}\right)+T\left(r, \frac{1}{P_{n}(f(q z+c))}\right)+S_{1}(r, f) \\
& \leq T(r, F)+(n+1) T(r, f)+S_{1}(r, f) .
\end{aligned}
$$

Then it follows from (7) and (8) that

$$
(m-n) T(r, f)+S_{1}(r, f) \leq T(r, F) \leq(n+m+1) T(r, f)+S_{1}(r, f) .
$$

We have by (9) that $S_{1}(r, F)=S_{1}(r, f)$. Similarly, we have $S_{1}(r, G)=S_{1}(r, g)$ and

$$
(m-n) T(r, g)+S_{1}(r, g) \leq T(r, G) \leq(n+m+1) T(r, g)+S_{1}(r, g) .
$$

Since $f(z)$ and $g(z)$ are entire functions of order zero and share $p(z) C M$, we have

$$
\frac{F(z)-p(z)}{G(z)-p(z)}=\eta
$$

where $\eta$ is a non-zero constant. If $\eta=1$, then we have $F(z)=G(z)$, that is, $f(z)^{m} P_{n}(f(q z+$ c)) $f^{\prime}(z)=g(z)^{m} P_{n}(g(q z+c)) g^{\prime}(z)$. 
If $\eta \neq 1$, then we have

$$
F(z)-\eta G(z)=p(z)(1-\eta)
$$

Since $P_{n}(z)$ has $t_{n}$ distinct zeros, by using the second main theorem and Lemma 2.2, we have

$$
\begin{aligned}
T(r, F) \leq & \bar{N}(r, F)+\bar{N}\left(r, \frac{1}{F}\right)+\bar{N}\left(r, \frac{1}{F-p(z)(1-\eta)}\right)+S(r, F) \\
\leq & \bar{N}\left(r, \frac{1}{P_{n}(f(q z+c))}\right)+\bar{N}\left(r, \frac{1}{f^{\prime}}\right)+N\left(r, \frac{1}{f}\right)+\bar{N}\left(r, \frac{1}{G}\right)+S_{1}(r, f) \\
\leq & \sum_{j=1}^{t_{n}} \bar{N}\left(r, \frac{1}{f(q z+c)-\gamma_{j}}\right)+\bar{N}\left(r, \frac{1}{f^{\prime}}\right)+N\left(r, \frac{1}{f}\right)+S_{1}(r, f) \\
& +\sum_{j=1}^{t_{n}} \bar{N}\left(r, \frac{1}{g(q z+c)-\gamma_{j}}\right)+\bar{N}\left(r, \frac{1}{g^{\prime}}\right)+N\left(r, \frac{1}{g}\right)+S_{1}(r, g) \\
\leq & \left(t_{n}+2\right) T(r, f)+\left(t_{n}+2\right) T(r, g)+S_{1}(r, f)+S_{1}(r, g),
\end{aligned}
$$

where $\gamma_{1}, \gamma_{2}, \ldots, \gamma_{t_{n}}$ are the distinct zeros of $P_{n}(z)$. Similarly, we have

$$
T(r, G) \leq\left(t_{n}+2\right) T(r, f)+\left(t_{n}+2\right) T(r, g)+S_{1}(r, f)+S_{1}(r, g) .
$$

Then (9), (10), (13), and (14) result in

$$
(m-n)(T(r, f)+T(r, g)) \leq 2\left(t_{n}+2\right)(T(r, f)+T(r, g))+S_{1}(r, f)+S_{1}(r, g),
$$

which contradicts $m \geq n+2 t_{n}+5$.

This completes the proof of Theorem 1.10.

\section{Competing interests}

The authors declare that they have no competing interests.

\section{Authors' contributions}

All authors contributed equally to the writing of this paper. All authors drafted the manuscript, read, and approved the final manuscript.

\section{Author details}

${ }^{1}$ Institute of Mathematics and Information Science, Jiangxi Normal University, Nanchang, 330022, China. ${ }^{2}$ Department of Informatics and Engineering, Jingdezhen Ceramic Institute, Jingdezhen, 333403, China.

\section{Acknowledgements}

This work was supported by the National Natural Science Foundation of China $(11301233,61202313,11171119)$, the Natural Science Foundation of Jiangxi Province in China (20132BAB211001) and Sponsored Program for Cultivating Youths of Outstanding Ability in Jiangxi Normal University of China, and the Foundation of Education Department of Jiangxi (GJJ14271, GJJ14644) of China.

\section{Received: 12 April 2014 Accepted: 30 June 2014 Published: 19 Aug 2014}

\section{References}

1. Hayman, WK: Meromorphic Functions. The Clarendon Press, Oxford (1964)

2. Yang, L: Value Distribution Theory. Springer, Berlin (1993)

3. Yi, HX, Yang, CC: Uniqueness Theory of Meromorphic Functions. Kluwer Academic, Dordrecht (2003). Chinese original, Science Press, Beijing, 1995

4. Chern, PTY: On meromorphic functions with finite logarithmic order. Trans. Am. Math. Soc. 358(2), 473-489 (2006) 
5. Xu, JF, Zhang, XB: The zeros of difference polynomials of meromorphic functions. Abstr. Appl. Anal. 2012, Article ID $357203(2012)$

6. Gross, F: On the distribution of values of meromorphic functions. Trans. Am. Math. Soc. 131, 199-214 (1968)

7. Hayman, WK: Picard values of meromorphic functions and their derivatives. Ann. Math. 70(2), 9-42 (1959)

8. Mues, E: Über ein Problem von Hayman. Math. Z. 164(3), 239-259 (1979)

9. Chen, HH, Fang, ML: On the value distribution of $f^{n} f^{\prime}$. Sci. China Ser. A 38, 789-798 (1995)

10. Chiang, YM, Feng, SJ: On the Nevanlinna characteristic of $f(z+\eta)$ and difference equations in the complex plane. Ramanujan J. 16, 105-129 (2008)

11. Halburd, RG, Korhonen, RJ: Difference analogue of the lemma on the logarithmic derivative with applications to difference equations. J. Math. Anal. Appl. 314, 477-487 (2006)

12. Halburd, RG, Korhonen, RJ: Nevanlinna theory for the difference operator. Ann. Acad. Sci. Fenn., Math. 31, 463-478 (2006)

13. Chen, ZX: On value distribution of difference polynomials of meromorphic functions. Abstr. Appl. Anal. 2011, Article ID $239853(2011)$

14. Laine, I, Yang, CC: Value distribution of difference polynomials. Proc. Jpn. Acad., Ser. A, Math. Sci. 83, 148-151 (2007)

15. $\mathrm{Xu}, \mathrm{HY}$ : On the value distribution and uniqueness of difference polynomials of meromorphic functions. Adv. Differ. Equ. 2013, Article ID 90 (2013)

16. Zheng, XM, Chen, ZX: On the value distribution of some difference polynomials. J. Math. Anal. Appl. 397(2), 814-821 (2013)

17. $\mathrm{Xu}, \mathrm{HY}$, Liu, BX, Tang, KZ: Some properties of meromorphic solutions of systems of complex $q$-shift difference equations. Abstr. Appl. Anal. 2013, Article ID 680956 (2013)

18. $\mathrm{Xu}, \mathrm{HY}, \mathrm{Tu}, \mathrm{J}, \mathrm{Zheng}, \mathrm{XM}$ : Some properties of solutions of complex $q$-shift difference equations. Ann. Pol. Math. 108(3), 289-304 (2013)

19. Zheng, $\mathrm{XM}, \mathrm{Xu}, \mathrm{HY}$ : On the deficiencies of some differential-difference polynomials. Abstr. Appl. Anal. 2014, Article ID 378151 (2014)

20. Zhang, JL, Korhonen, RJ: On the Nevanlinna characteristic of $f(q z)$ and its applications. J. Math. Anal. Appl. 369 537-544 (2010)

21. Xu, JF, Zhang, XB: The zeros of $q$-difference polynomials of meromorphic functions. Adv. Differ. Equ. 2012, Article ID $200(2012)$

22. Liu, K, Qi, XG: Meromorphic solutions of q-shift difference equations. Ann. Pol. Math. 101, 215-225 (2011)

10.1186/1029-242X-2014-295

Cite this article as: Zheng and $\mathrm{Xu}$ : On value distribution and uniqueness of meromorphic function with finite logarithmic order concerning its derivative and q-shift difference. Journal of Inequalities and Applications 2014, 2014:295

\section{Submit your manuscript to a SpringerOpen ${ }^{\ominus}$ journal and benefit from:}

- Convenient online submission

Rigorous peer review

- Immediate publication on acceptance

- Open access: articles freely available online

- High visibility within the field

- Retaining the copyright to your article 